\title{
Identification of Three Type II Toxin-Antitoxin Systems in Streptococcus suis Serotype 2
}

\author{
Jiali Xu ${ }^{1}$, Nian Zhang ${ }^{1}$, Manman Cao ${ }^{1}$, Sujing Ren ${ }^{1}$, Ting Zeng ${ }^{1}$, Minglu Qin ${ }^{1}$, Xigong Zhao ${ }^{1}$, \\ Fangyan Yuan ${ }^{2}$, Huanchun Chen ${ }^{1}$ and Weicheng Bei ${ }^{1, *}$ \\ 1 State Key Laboratory of Agricultural Microbiology, The Cooperative Innovation Center for Sustainable Pig \\ Production, College of Veterinary Medicine, Huazhong Agricultural University, Wuhan 430070, China; \\ jialixu@webmail.hzau.edu.cn (J.X.); nianzhang@webmail.hzau.edu.cn (N.Z.); \\ mmcao@webmail.hzau.edu.cn (M.C.); rensujing0327@163.com (S.R.); tingzeng@webmail.hzau.edu.cn (T.Z.); \\ mlqin199591@126.com (M.Q.); xgzhao@webmail.hzau.edu.cn (X.Z.); chenhch@mail.hzau.edu.cn (H.C.) \\ 2 Key Laboratory of Prevention and Control Agents for Animal Bacteriosis, Institute of Animal Husbandry \\ and Veterinary Sciences, Hubei Academy of Agricultural Sciences, Wuhan 430064, China; \\ fangyanyuan12@163.com \\ * Correspondence: beiwc@mail.hzau.edu.cn; Tel.: +86-27-8728-8629
}

Received: 22 October 2018; Accepted: 7 November 2018; Published: 13 November 2018

\begin{abstract}
Type II toxin-antitoxin (TA) systems are highly prevalent in bacterial genomes and have been extensively studied. These modules involve in the formation of persistence cells, the biofilm formation, and stress resistance, which might play key roles in pathogen virulence. SezAT and yefM-yoeB TA modules in Streptococcus suis serotype 2 (S. suis 2) have been studied, although the other TA systems have not been identified. In this study, we investigated nine putative type II TA systems in the genome of $S$. suis 2 strain SC84 by bioinformatics analysis and identified three of them (two relBE loci and one parDE locus) that function as typical type II TA systems. Interestingly, we found that the introduction of the two RelBE TA systems into Escherichia coli or the induction of the ParE toxin led to cell filamentation. Promoter activity assays indicated that RelB1, RelB2, ParD, and ParDE negatively autoregulated the transcriptions of their respective TA operons, while RelBE2 positively autoregulated its TA operon transcription. Collectively, we identified three TA systems in S. suis 2, and our findings have laid an important foundation for further functional studies on these TA systems.
\end{abstract}

Keywords: RelBE; ParDE; cell filamentation; autoregulation

Key Contribution: Three type II TA systems (two RelBE and one ParDE) were identified in S. suis 2. The introduction of two RelBE into E. coli or the induction of ParE led to cell filamentation, and RelB1, RelB2, ParD, and ParDE negatively autoregulated transcriptions of their respective TA operons, while RelBE2 positively autoregulated its TA operon transcription.

\section{Introduction}

Streptococcus suis (S. suis) is an important major swine and zoonotic pathogen that causes severe infection [1-3]. It is associated with a variety of serious diseases, including arthritis, septicemia, pneumonia, endocarditis, and meningitis in pigs and leads to great economic losses worldwide $[4,5]$. S. suis infection in humans causes arthritis, septicemia, meningitis and streptococcal toxic shock syndrome (STSS) through direct contact with sick pigs or pork by-products [6,7]. According to the composition of capsular polysaccharide (CPS), 33 serotypes (types 1 to 31, 33, and 1/2) in S. suis have been described [8]. Among them, serotype 2 is acknowledged as the most prevalent and virulent 
serotype, and it has been frequently isolated from diseased pigs [2]. Two large outbreaks of human S. suis 2 infection occurred in 1998 and 2005 in China, causing great public concern due to the high pathogenicity of this zoonotic pathogen in humans [9]. However, the pathogenesis of S. suis 2 infection has not been well understood yet. Previously, we have predicted 9 type II Toxin-antitoxin (TA) systems in the genome of S. suis SC84 by bioinformatics analysis [10]. The yefM-yoeB TA system in S. suis 2 has been described, but this does not contribute to virulence in the murine infection model [10]. The SezAT module has firstly been characterized as an active TA system in S. suis 2 strain 05ZYH33, which promotes the maintenance of the SsPI-1 pathogenicity island [11], yet its function remains unclear. First and foremost, other TA systems, prevalent in S. suis 2, should be identified, and their functions should be explored. This would provide further insight into the mechanisms of severe $S$. suis 2 infection.

Recently, type II TA systems, in which toxins and antitoxins are proteins, have been widely prevalent in bacterial genomes and the most extensively studied [12]. Based on the sequence similarity and nature of toxins, several super-families of type II TA modules have been defined [13,14]. Multiple studies have reported some super-families of type II TA systems, including MazEF, RelBE, HigBA, HipBA, VapBC, Phd/Doc, CcdAB, HicAB and ParDE [15-19]. MazEF in Escherichia coli $[18,20]$, Mycobacterium tuberculosis [21], Staphylococcus equorum [22] and Streptococcus mutans [23] has been well characterized. The role of RelBE, as one of the most common type II systems, in important physiological processes of Vibrio cholerae has been described [14]. Seventeen TA pairs have been identified in V. cholera, of which, seven RelBE, three ParDE, two HigBA, and a single Phd/Doc were well characterized [14,24-26]. The hipBA locus in M.tuberculosis, as a ubiquitous TA locus, has been found to be involved in stress response [27]. MazEF, VapBC and Phd/Doc, the three putative TA modules of Mycobacterium smegmatis, play an essential role in cell survival [28]. Moreover, type II TA systems have been found to contribute to the virulence of Salmonella [29] and Leptospira interrogans [30]. There is increasing evidence that type II TA systems are ubiquitous and strongly related to the formation of persistence cells, the regulation of biofilm formation, stress resistance, and other biological processes, and that they contribute to bacterial pathogenicity $[13,15,31,32]$. Thus, the identification of novel type II TA systems in pathogenic microbes, especially S. suis 2, is urgently needed.

This study identified three active type II TA systems in S. suis 2, including two RelBE and one ParDE. A total of five TA systems, present in S. suis 2, have been found and described for the first time. Our results have provided new information for further functional studies on these TA systems.

\section{Results}

\subsection{Identification of Putative Type II TA Systems in S. suis 2}

The putative type II TA loci in S. suis SC84 were predicted with TAfinder, a newly developed online tool in TADB (Toxin-Antitoxin Database, http:/ / 202.120.12.135/TADB2/Introduction.html\#table_s2), which can quickly detect the TA prediction [12]. Nine putative type II TA systems are shown in Table 1 and distributed widely in the circular complete genome map (Figure S1). Both the nine putative TA systems and the ID numbers of each putative toxin or antitoxin are included in the Figure S1. The genetic organization of putative type II TA systems are shown in Figure S2. Each antitoxin gene is located upstream of the toxin gene, except for the putative TA_2, which is associated with the direction of the gene encoding (Figure S2). It is interesting that TA_2 and TA_3 were found to share the same toxin gene, according to the predicted result, which may need to be further researched. Additionally, the physical distance (in bp) between the putative antitoxin and toxin coding sequences, TA protein domain pair, and TA family (based on the toxin protein) are shown in Table 1. While the TA_4 system was found to be entirely homologous to SezAT in S. suis 05ZYH33 [11], suggesting that TA_4 works as typical type II TA systems, TA_8 was identified as the yefM-yoeB system in S. suis SC84 [10]. Therefore, the remaining seven modules, including five modules belonging to the $\mathrm{relBE} / \mathrm{parDE}$ family, were regarded as putative type II TA systems and chosen for further study. 
Table 1. Putative type II toxin-antitoxin (TA) systems predicted in S. suis SC84.

\begin{tabular}{ccccccc}
\hline TA_No. & Toxin & Antitoxin & Strand & Distance(bp) $^{\mathbf{1}}$ & Domain Pair $^{2}$ & Family $^{\text {Dard }}$ \\
\hline TA_1 & SSUSC84_0548 & SSUSC84_0547 & + & -11 & RHH-RelE & relBE/parDE \\
TA_2 & SSUSC84_0791 & SSUSC84_0790 & - & 38 & - & - \\
TA_3 & SSUSC84_0791 & SSUSC84_0792 & - & -17 & RHH-RelE & relBE/parDE \\
TA_4 & SSUSC84_0841 & SSUSC84_0842 & - & -1 & - & SezAT \\
TA_5 & SSUSC84_0861 & SSUSC84_0860 & + & 14 & Xre-MNT & relBE/parDE \\
TA_6 & SSUSC84_1034 & SSUSC84_1035 & - & 201 & - & - \\
TA_7 & SSUSC84_1348 & SSUSC84_1349 & - & 1 & RHH-RelE & relBE/parDE \\
TA_8 & SSUSC84_1817 & SSUSC84_1818 & - & 1 & PHD-RelE & yefM-yoeB \\
TA_9 & SSUSC84_1820 & SSUSC84_1821 & - & -11 & RHH-RelE & relBE/parDE \\
\hline
\end{tabular}

${ }^{1}$ The distance (bp) indicates the physical distance (in bp) between the putative antitoxin and toxin coding sequences, and the toxin and antitoxin genes are overlapped $(-)$ or separated $(+)$ by a few nucleotides; ${ }^{2}$ A domain pair represents the TA protein domain pair of each antitoxin and its cognate toxin. -, it means no TA domain pair is found.

\subsection{Each Putative Type II TA Locus Was Encoded by An Operon}

The two genes of the TA module were organized into an operon, and the TA module was verified by RT-PCR analysis. Therefore, the total RNA was extracted from S. suis 2 and used to synthesize cDNAs. Then, cDNAs were PCR amplified using the assigned primer pairs, which anneals to the $5^{\prime}$-end and $3^{\prime}$-region of the coding sequence of each putative TA locus. The result of RT-PCR analysis (Figure 1) showed that the expected sizes of PCR products were in line with those of the genomic DNA (gDNA). No PCR products were detected in the negative controls (cDNA-), in which the reverse transcription operated without the reverse transcriptase, therefore eliminating genomic DNA contamination. These data indicated that, for each putative TA module, the toxin genes and antitoxin genes were actively co-transcribed and organized into a bicistronic operon.

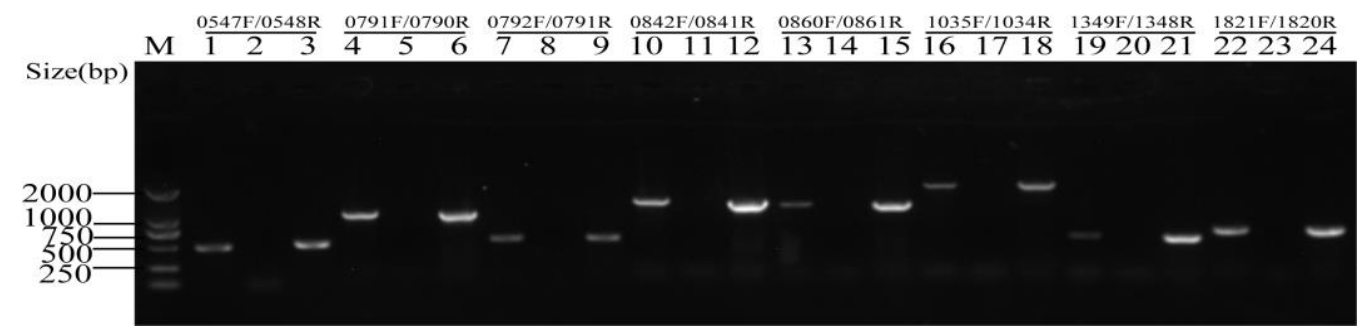

Figure 1. Co-transcription analysis of putative type II toxin-antitoxin (TA) modules in S. suis 2. The total RNA was isolated from the logarithmic phase $S$. suis 2 and used to synthesize cDNAs. PCR was carried out, with primer pairs indicated above the lanes. Lanes 1, 4, 7, 10, 13, 16, 19 and 22 represent the amplification using cDNAs as the template; Lanes 2, 5, 8, 11, 14, 17, 20 and 23 represent the amplification using CDNA- (RNA converted into a cDNA reaction without reverse transcriptase) as the template; and lanes $3,6,9,12,15,18,21$ and 24 represent the amplification using genomic DNA (gDNA) as the template. Lane M indicates the DL2000 DNA Marker.

\subsection{Effects of Each Putative TA System on the Growth of E. coli Using the Selective Expression Vector pETBAD}

Primarily, we chose the selective expression vector, pETBAD [10], to determine whether the putative TA systems could typically be described as the phenomenon of toxins that are toxic to E. coli being susceptible of neutralization by their cognate antitoxins. The plasmid of pETBAD was generated in order to independently control the expression of toxins by arabinose or the expression of antitoxins by isopropyl $\beta$-D-thiogalactopyranoside (IPTG). The plasmids of the pETBAD-antitoxin-toxin (pETBAD-0547-0548, -0790-0791, -0792-0791, -0842-0841, -0860-0861, -1035-1034, -1349-1348, -1818-1817 and -1821-1820) and pETBAD were transformed into E. coli BL21 (DE3) pLysS cells, respectively. BL21 (DE3) pLysS cells, harboring the constructed plasmids of pETBAD-0547-0548, -0842-0841, $-1349-1348,-1818-1817$, and -1821-1820, exhibited a significant growth inhibition, compared to those 
containing pETBAD, with increasing concentrations of L-arabinose. BL21 (DE3) pLysS cells, harboring the constructed plasmids of pETBAD-0790-0791, -0792-0791, -0860-0861, and -1035-1034, exhibited almost identical growth, compared to those containing the pETBAD plasmid, under the same conditions (Figure 2A-D). However, no major difference in E. coli growth was observed between the co-induction of BL21 (DE3) pLysS cells, harboring the constructed plasmids of the pETBAD-antitoxin-toxin (pETBAD-0547-0548, -0790-0791, -0792-0791, -0842-0841, -0860-0861, -1035-1034, -1349-1348, -1818-1817 and -1821-1820) and pETBAD (Figure 2F), and the induction of each putative antitoxin alone (Figure 2E), which was almost consistent with the E. coli growth of the control group (Figure 2G). Our results indicated that the loci, TA_1, TA_4, TA_7, TA_8 and TA_9, potentially work as typical type II TA systems. As TA_4 and TA_8 have been studied previously $[10,11]$, we carried out the following experiments to further confirm the toxic effects of other TA systems.

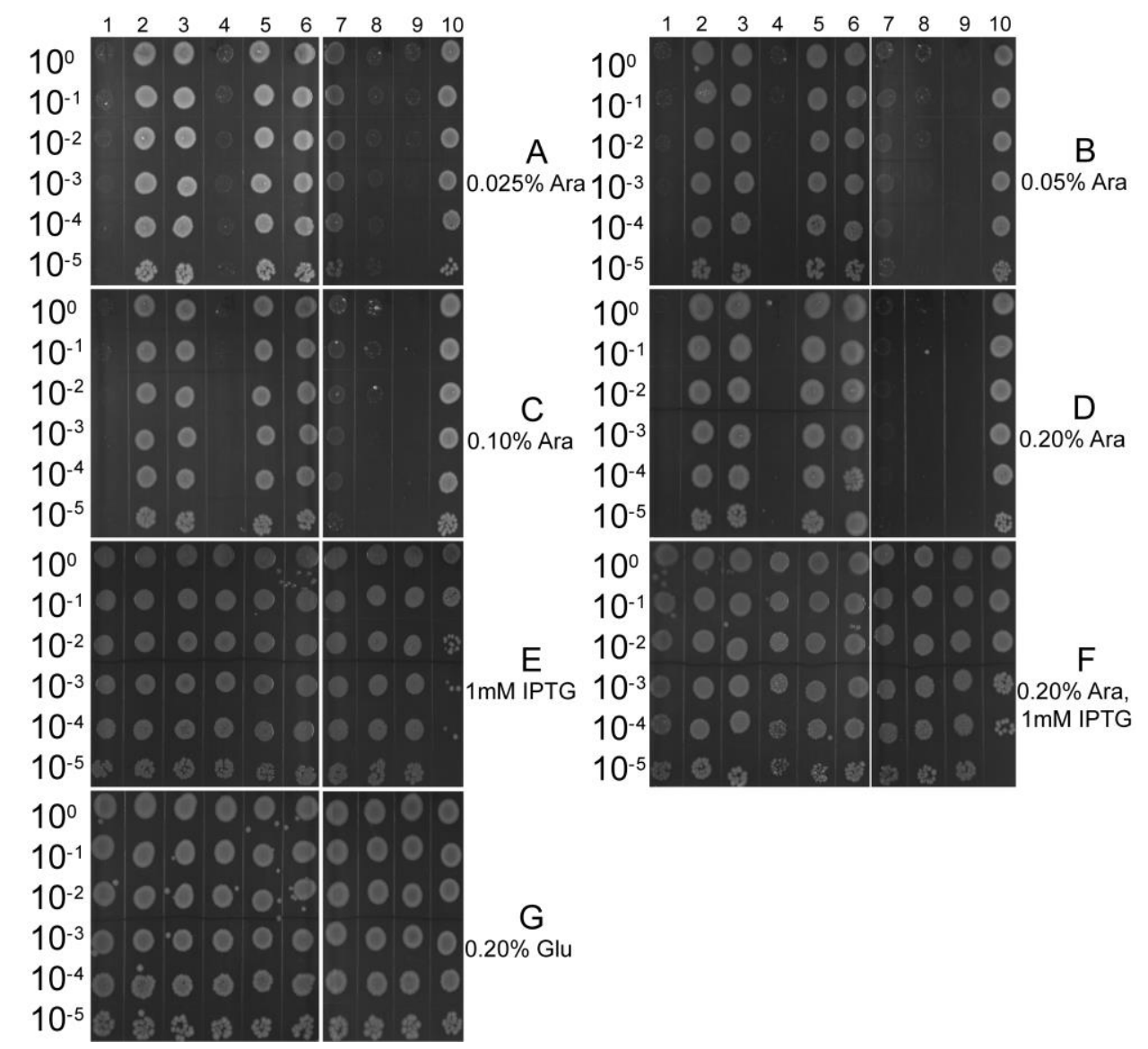

Figure 2. Effect of the induction of nine putative toxin-antitoxin (TA) modules, cloned separately into the selective expression vector, on E. coli growth. E. coli BL21 (DE3) pLysS cells, harboring the constructed pETBAD-0547-0548 (Lane 1), -0790-0791 (Lane 2), -0792-0791 (Lane 3), -0842-0841 (Lane 4), -0860-0861 (Lane 5), -1035-1034 (Lane 6), -1349-1348 (Lane 7), -1818-1817 (Lane 8), -1821-1820 (Lane 9), and pETBAD (Lane 10), were grown to $\mathrm{OD}_{600}$ of $0.6-0.8$. Each culture was serially diluted, and $5-\mu \mathrm{L}$ drops, with the concentration ranging from $10^{0}$ (top) to $10^{-5}$ (bottom), were respectively spotted onto the plate, with different inducers. (A-D) $0.025 \%, 0.05 \%, 0.10 \%$, and $0.20 \%$ L-arabinose were added successively to induce expression of putative toxins; (E) $1 \mathrm{mM}$ isopropyl $\beta$-D-thiogalactopyranoside (IPTG) was added to induce the expression of putative antitoxins; (F) Both $0.20 \%$ L-arabinose and $1 \mathrm{mM}$ IPTG were added to induce the expression of putative TA modules; $(G) 0.20 \%$ D-glucose (repression conditions) was added as the control. 


\subsection{Evaluation of the Toxic Effects of Putative Toxins on the Growth of E. coli}

The pBADhisA plasmid was used to determine whether the putative toxins were toxic to E. coli, and we cloned the putative toxin genes into the pBADhisA expression vector. TA_2 and TA_3 had the same toxin gene, SSUSC84_0791. Therefore, the six plasmids of the pBADhisA-toxin (pBADhisA-0548, $-0791,-0861,-1034,-1348$, and -1820) and pBADhisA were transformed into E. coli Top10 cells. In the presence of $0.20 \%$ D-glucose (repression conditions), Top10 cells, harboring the plasmid of pBADhisA-0548 demonstrated a moderate growth defect, compared to those harboring the plasmid of pBADhisA, while Top10 cells, harboring the plasmids of pBADhisA-0791, $-0861,-1034,-1348$, and -1820 , exhibited no major difference in E. coli growth (Figure 3A). Under induction conditions, Top10 cells, containing pBADhisA-0791 and pBADhisA-1034, exhibited no growth inhibition, compared to those containing pBADhisA. Under the same induction conditions, Top10 cells, carrying pBADhisA-0548, $-0861,-1348$, and -1820 , exhibited growth inhibition, unlike those carrying pBADhisA (Figure 3B).

A

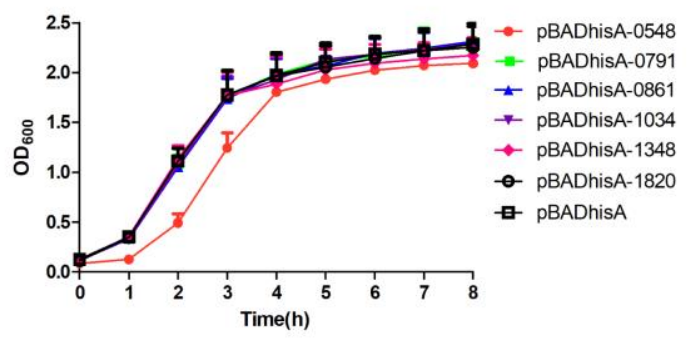

C

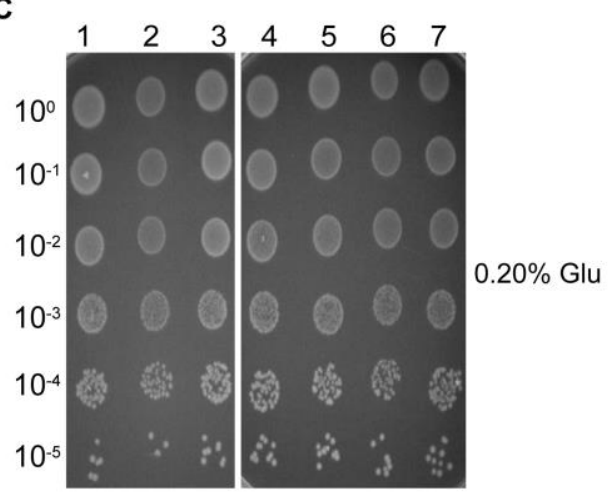

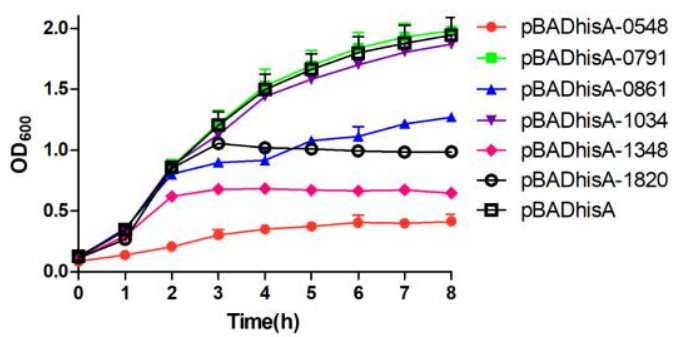

D

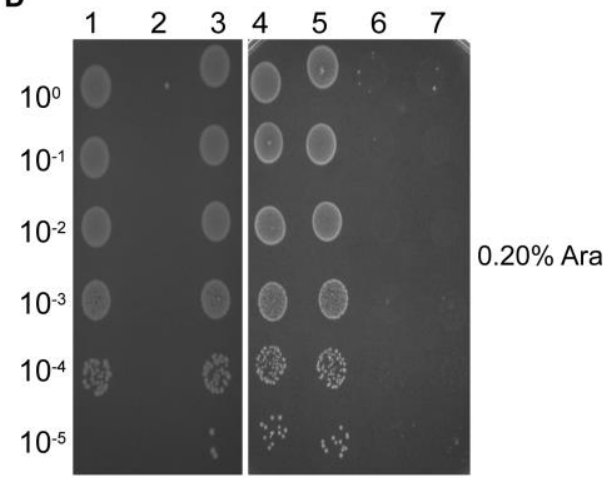

Figure 3. Effect of the induction of putative toxins on the growth of E. coli. E. coli Top10 cells, harboring the corresponding plasmids, were grown to $\mathrm{OD}_{600}$ of $0.05-0.20$. Each culture was then divided into two equal volumes and supplemented with either $0.20 \%$ D-glucose (A) or $0.20 \%$ L-arabinose (B). Culture growth was monitored by measuring $\mathrm{OD}_{600}$ every hour. Growth curves are representative of at least 3 independent experiments. Top10 cells, harboring the corresponding pBADhisA-0548 (Lane 2), -0791 (Lane 3), -0861 (Lane 4), -1034 (Lane 5), -1348 (Lane 6), -1820 (Lane 7), and pBADhisA (Lane 1), were grown to $\mathrm{OD}_{600}$ of $0.6-0.8$. Each culture was serially diluted, and 5- $\mu \mathrm{L}$ drops, with the concentration ranging from of $10^{0}$ (top) to $10^{-5}$ (bottom), were successively spotted onto the different plates with (C) $0.20 \%$ D-glucose or (D) $0.20 \%$ L-arabinose.

Overnight cultures of E. coli Top10 cells, carrying the plasmids of pBADhisA-0548, -0791, -0861, $-1034,-1348,-1820$ or pBADhisA (control), were diluted at 1:100 in LB-ampicillin, grown to OD 600 of $0.6-0.8$ and serially diluted, and $5-\mu \mathrm{L}$ drops were spotted onto the different plates with $0.20 \%$ D-glucose or $0.20 \%$ L-arabinose. As shown in Figure 3C,D, the Top10 cells, carrying pBADhisA-0548, -1348 and -1820 (toxin genes of TA_1, TA_7, and TA_9) on the plate with $0.20 \%$ L-arabinose, exhibited obvious growth inhibition, while the Top10 cells, carrying pBADhisA-0791, -0861, and -1034, exhibited normal growth (Figure 3D). By contrast, no major difference in growth, between Top10 cells, carrying 
pBADhisA-0548, $-0791,-0861,-1034,-1348$, and -1820 , and those carrying pBADhisA on the plate with $0.20 \%$ D-glucose was observed (Figure 3C). These results indicated that TA_1, TA_7, and TA_9 are potential type II TA systems.

\subsection{Evaluation of the Antitoxic Effects of Putative Antitoxins}

To determine whether the toxic effect of each toxin can be alleviated by its cognate antitoxin, the sequence of each putative antitoxin was cloned separately into the pET30a plasmid. Additionally, the resulting plasmids were designated as pET30a-antitoxin (pET30a-0547, -1349, and -1821). E. coli BL21 (DE3) pLysS cells, harboring pBADhisA-0548 and pET30a-0547 (or pET30a, as control), pBADhisA-1348 and pET30a-1349 (or pET30a, as control), and pBADhisA-1820 and pET30a-1821 (or pET30a, as control), were successfully obtained by co-transformation and selection with ampicillin and kanamycin. When BL21 (DE3) pLysS cells, harboring these plasmids, were grown to $\mathrm{OD}_{600}$ of $0.20-0.30$, each culture was supplemented with $0.20 \%$ L-arabinose and $1 \mathrm{mM}$ IPTG. The induction of TA_1 (Figure 4A), TA_7 (Figure 4B), and TA_9 (Figure 4C) resulted in normal growth, while BL21 (DE3) pLysS cells, carrying both pBADhisA-toxin and pET30a plasmids, demonstrated a significant growth inhibition (Figure 4A-C), suggesting that the putative antitoxins of TA_1, TA_7, and TA_9 could neutralize the cognate toxin.
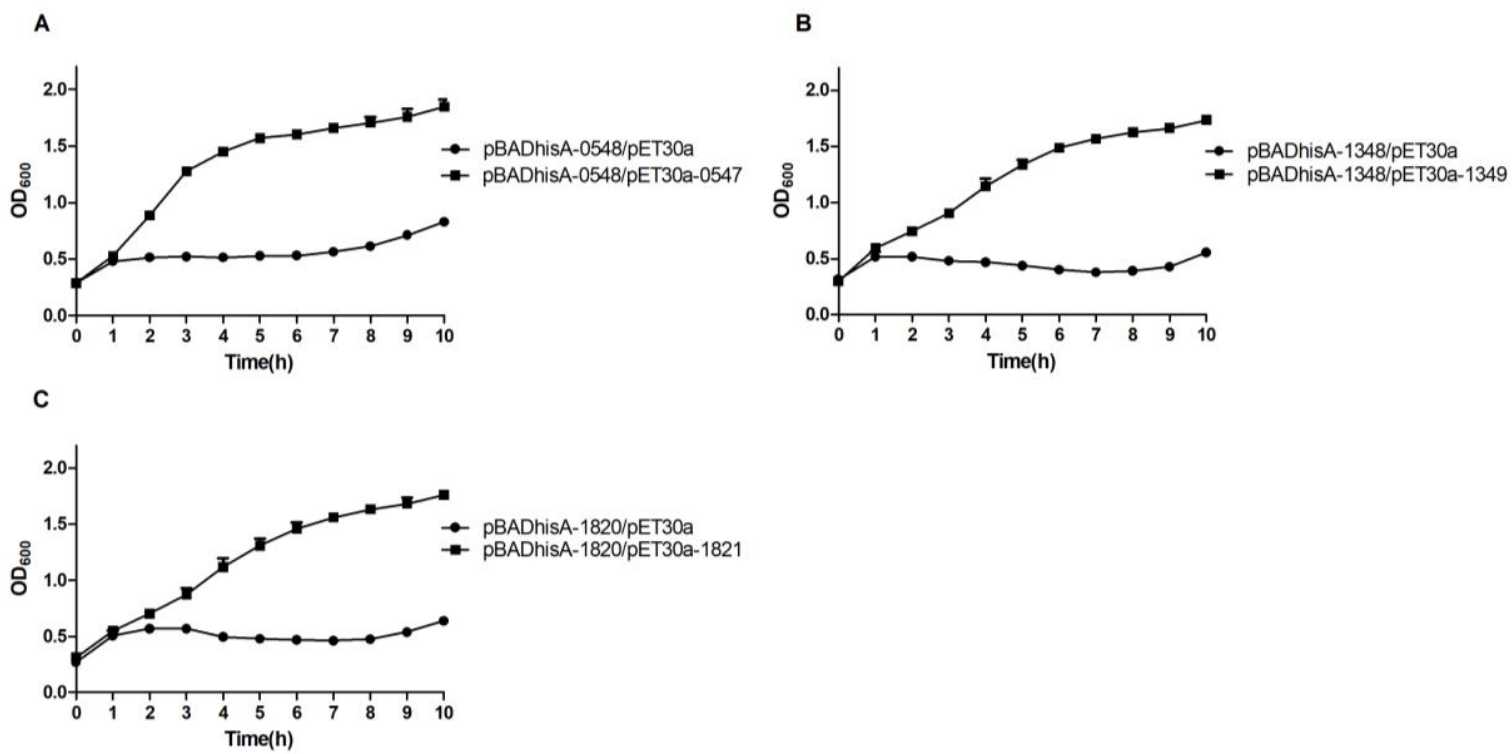

Figure 4. Effect of the co-expression of each putative antitoxin and its cognate toxin on the growth of E. coli. BL21 (DE3) pLysS cells, harboring the constructed (A) pBADhisA-0548 and pET30a-0547 (or pET30a, as control); (B) pBADhisA-1348 and pET30a-1349 (or pET30a, as control); and (C) pBADhisA-1820 and pET30a-1821 (or pET30a, as control), which were grown to $\mathrm{OD}_{600}$ of $0.2-0.3$. Each culture was supplemented with both $0.20 \% \mathrm{~L}$-arabinose and $1.00 \mathrm{mM}$ isopropyl $\beta$-D-thiogalactopyranoside (IPTG). Culture growth was evaluated by measuring $\mathrm{OD}_{600}$ every hour. Growth curves are representative of at least 3 independent experiments.

Taken together, these above results indicated that TA_1, TA_7, and TA_9 loci work as typical type II TA systems.

During these growth experiments, microscopic examination showed that normal-sized cells become filamentous after the induction of TA_1, TA_7, and TA_9 systems (Figure 5A-C). The TA_1 and TA_7 systems were introduced into E. coli and caused cell filamentation, with or without the inducers (IPTG, L-arabinose) at $5 \mathrm{~h}$. The induction of the whole TA_9 system or the toxin of TA_9 alone in E. coli exhibited cell filamentation, while no cell filamentation was observed in the absence of inducers (-IPTG, L-arabinose) (Figure 5C). Therefore, the introduction of TA_1 and TA_7 systems 
into E. coli led to cell filamentation. Additionally, the induction of the toxin of TA_9 caused more significant cell filamentation, compared that of the whole TA_9 system. It was reported that the cell morphology, induced by the toxin, ParE, was filamentous in E. coli [33] and Caulobacter crescentus [34]. After confirming that TA_1, TA_7, and TA_9 loci work as typical type II TA systems, the toxin of TA_1 was renamed RelE1, the toxin of TA_7 was renamed RelE2, and the toxin of TA_9 was renamed ParE. The antitoxins of TA_1, TA_7, and TA_9 were respectively renamed RelB1, RelB2, and ParD.
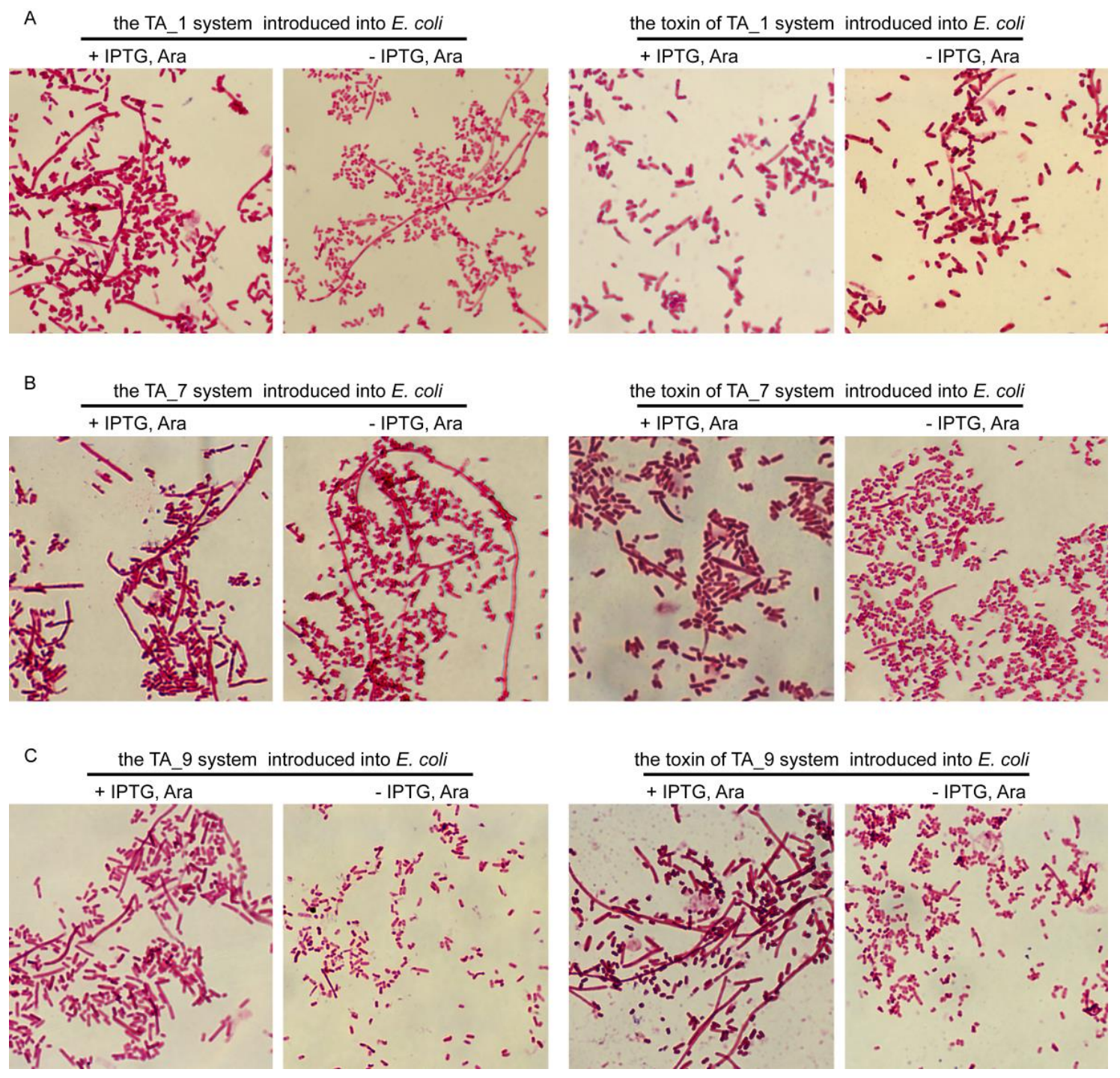

Figure 5. Introduction of the S. suis toxin or toxin-antitoxin (TA) complex into E. coli leads to cell filament formation. (A) The TA_1 system or the toxin of the TA_1 system, (B) the TA_7 system or the toxin of the TA_7 system, and (C) the TA_9 system or the toxin of the TA_9 system were introduced into E. coli BL21 (DE3) pLysS cells and were induced with $1 \mathrm{mM}$ isopropyl $\beta$-D-thiogalactopyranoside (IPTG) and $0.20 \%$ L-arabinose (+IPTG, Ara) and without IPTG and L-arabinose (-IPTG, Ara) (as control). Light microscope morphology of E. coli cells, using Gram staining $(\times 100)$, was performed at $5 \mathrm{~h}$ after induction.

\subsection{Antitoxin or TA Complex Autoregulates the TA Operon}

In typical type II TA systems, the antitoxin alone or the TA complex binds to the promoter and regulates the transcription of the TA operon [35]. The $\beta$-galactosidase activity was measured in order to study the autoregulation of the three TA (relBE1, relBE2, and parDE) operons, as previously described [36,37]. The plasmids of pHGEI01-antitoxin' (-relB1', -relB2', and -parD'), 
pHGEI01-antitoxin-toxin' (-relB1-relE1', -relB2-relE2', and -parD-parE'), and pHGEI01-antitoxin-toxin (relB1-relE1, -relB2-relE2, and -parD-parE) were constructed and transformed into E. coli WM3064, respectively. As for the RelBE1 system, the promoter activity in WM3064 cells, carrying the pHGEI01-relB1' plasmid, was significantly higher than that in cells carrying pHGEI01-relB1-relBE1' $(p=0.0072)$ (Figure 6A), indicating that the antitoxin, RelB1, repressed the promoter activity. However, it is a pity that we failed to construct the pHGEI01-relB1-relE1 after several attempts. As for the RelBE2 system, the promoter activity was obviously repressed by the antitoxin, RelB2, but it was enhanced by the RelBE2 TA complex (Figure 6B). Concerning the ParDE system, it was found that the promoter activity in WM3064 cells, carrying the pHGEI01-parD' plasmid, was significantly higher than that in cells carrying pHGEI01-parD-parE' $(p=0.0048)$ and pHGEI01-parD-parE $(p<0.0001)$. Moreover, it was found that the promoter activity in WM3064 cells, carrying the pHGEI01-parD-parE' plasmid, was significantly higher than that in cells carrying pHGEI01-parD-parE $(p=0.0002)$ (Figure 6C), indicating that ParD and ParDE repressed the promoter activity. Additionally, it was confirmed that the ParDE complex repressed the promoter activity more significantly than ParD. Furthermore, concerning the RelBE2 system, the inhibitory effect of antitoxin, RelB2, on the transcription of the TA operon was reversed by its cognate toxin, RelE2. As for the ParDE system, the toxin ParE helped the antitoxin, ParD, to repress the promoter activity. Taken together, these results indicated that RelB1, RelB2, ParD, and ParDE negatively autoregulated the transcriptions of their respective TA operons, while RelBE2 positively autoregulated its TA operon transcription.

A

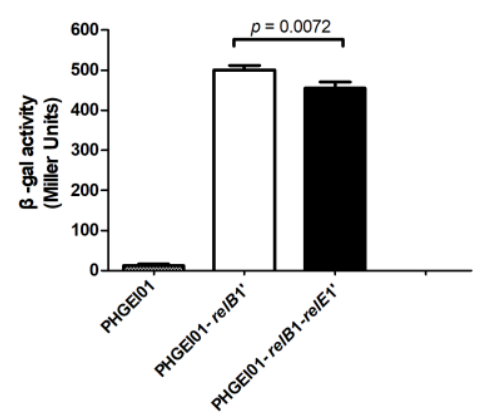

B

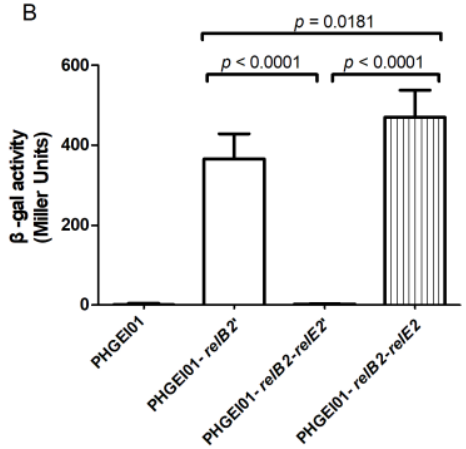

C

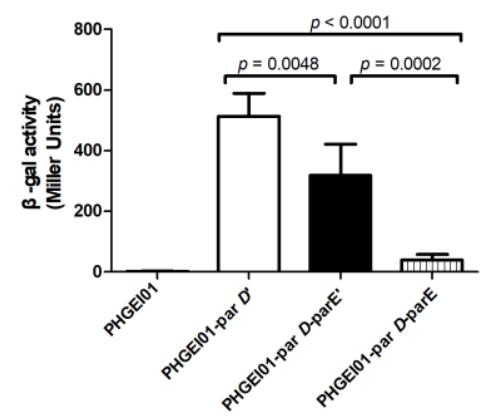

Figure 6. The antitoxin or TA complex autoregulates the TA operon. (A) The RelBE1 system; (B) The RelBE2 system; (C) The ParDE system. E. coli WM3064 cells, harboring the corresponding reporter plasmids, were collected in the mid-exponential phase $\left(\mathrm{OD}_{600} \sim 0.6-0.7\right)$ and tested for $\beta$-galactosidase activity. The descriptive data of the means \pm standard deviations for three of the independent experiments are shown. The statistical significance was tested using a one-tailed unpaired $t$ test. A $p<0.05$ was considered statistically significant.

\section{Discussion}

The type II TA modules are ubiquitous in various bacteria and archaea $[12,29,38,39]$ and have drawn worldwide attention in recent years. For example, the VapBC TA systems in M. tuberculosis $[40,41]$ and Streptomyces sp. [36] were described. RelBE, belonging to a new TA family, was reported in E. coli K-12 [42]. The yefM-yoeB locus in S. suis [10], Streptococcus pneumoniae [43], and Staphylococcus aureus [44,45] was described. SezAT in S. suis [11] and PezAT in S. pneumoniae [46-48] were described. Many type II TA systems in various important pathogens, especially $M$. tuberculosis $[27,40,41,49]$ and $S$. pneumoniae [43,46-48,50-52], which could lead to severe infection in humans, have been well studied.

In this study, we chose the TAfinder tool and found nine putative TA systems in S. suis SC84. We successfully constructed the related plasmids (pETBAD-antitoxin-toxin, pBADhisA-toxin, and pET30a-antitoxin) and identified three TA systems (two RelBE and one ParDE), which have previously been uncharacterized in S. suis SC84. The expression of SSUSC84_0791, SSUSC84_0861, and SSUSC84_1034 exhibited no major growth defect (Figure 2A-D and Figure 4B), suggesting 
that TA_2, TA_3, TA_5, and TA_6 loci may not work as active TA systems. However, the effect of SSUSC84_0861 induction on E. coli growth in the liquid medium (Figure 3B) was found to be inconsistent with that on the solid medium (Figure 2A-D and Figure 3D). It was not observed that the product of the gene, SSUSC84_0861, could inhibit the growth of E. coli on the solid medium (Figure 2A-D and Figure 3D). Therefore, it remains to be explored whether or not SSUSC84_0861 works as a toxin in the future. Therefore, the effect of the plasmid of the pETBAD-antitoxin-toxin alone on E. coli growth is equal to the effect of both the pBADhisA-toxin and pET30a-antitoxin, under the condition of the induction of the toxin and its cognate antitoxin in vitro. It is convenient to use the selective expression vector, pETBAD, to demonstrate the effect of toxin-antitoxin systems on E. coli growth [10].

Structural analysis indicates that the toxin, ParE, is homologous to RelE and YoeB toxins, but the cellular target of ParE is different from that of RelE [34]. The RelE protein cleaves mRNA in the ribosomal A-site to inhibit translation [53,54], while ParE has been distinguished by inhibiting DNA gyrase and thereby blocking DNA replication [33]. The yefM-yoeB has been fused with the relEB family, while YoeB exhibits a similar tertiary fold to RelE [55]. As the previous report found that the toxin,

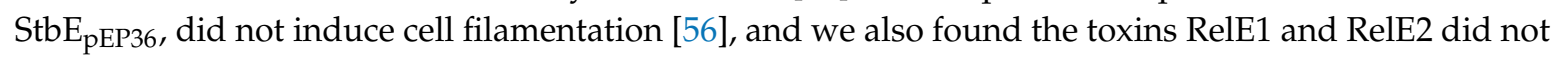
induce cell filamentation (Figure 5A,B). The mechanism by which the introduction of the two RelBE systems into E. coli leads to cell filamentation (Figure $5 \mathrm{~A}, \mathrm{~B}$ ), and the introduction of yefM-yoeB inhibits E. coli growth, under both the repression and induction conditions [10], remains to be explored in the future. Additionally, the induction of the toxin, ParE, led to cell filamentation in S. pneumoniae [51], while the induction of the ParD/ParE complex or ParE in E. coli exhibited different lengths of cells (Figure 5C), which may be consistent with the finding that the ParE toxin can inhibit cell division and that ParD prevents the inhibition by blocking the binding of ParE to gyrase and reducing cell damage [33]. The result that the ParD protein repressed the expression of the parDE operon of S. suis 2 is consistent with the function of ParD, as a transcriptional repressor in E. coli [57]. We also found that the ParDE complex repressed the promoter activity more significantly than ParD in S. suis 2 (Figure 6C), suggesting that the toxin, ParE, helped the antitoxin, ParD, to repress the promoter activity. However, the MParD2 repressed the promoter activity more strongly than MParDE2 (encoded by the parDE2 gene of $M$. tuberculosis) in the surrogate M. smegmatis [58]. These results indicated that ParD and ParDE negatively autoregulated the transcriptions of their respective TA operons. The ParDE TA system in other bacteria was well characterized [24,33,57-59], which might help to elucidate the functions of ParDE in S. suis 2 in further studies.

In E. coli, RelE was confirmed to enhance the repressor activity of RelB [53,60]. Additionally, the RelBE2sca complex or RelB2sca was found to repress the transcription of the TA operon in Streptomyces cattleya DSM46488 [61]. We failed to construct the pHGEI01-relB1-relE1 plasmid due to several base mutations. Therefore, it remains unclear how the RelBE1 complex regulates the transcription of the TA operon. There is a new finding that RelJ, RelBE, RelFG, and RelJK function as transcriptional repressors, and RelB and RelF function as transcriptional activators, by $\beta$-galactosidase activity analysis [49]. This study found, first, that the RelBE2 complex positively regulates the promoter activity (Figure 6B), while the RelBE of other bacteria negatively regulates the promoter activity $[49,53,60,61]$. Considering that type II TA systems contribute to the formation of persistence cells, stress response, biofilm formation and other various biological processes [13,15,31,62-64], the mechanism of three TA systems in S. suis 2 remains to be further explored in future studies.

In summary, we have identified two RelBE TA systems and one ParDE TA system in S. suis SC84. We also found that the introduction of the two RelBE TA systems into E. coli or the induction of the ParE toxin led to cell filamentation and that RelB1, RelB2, ParD, and ParDE negatively autoregulated the transcriptions of their respective TA operons, while RelBE2 positively autoregulated its TA operon transcription by promoter activity assays. However, the functions of three TA systems in S. suis 2 need to be intensively studied. 


\section{Materials and Methods}

\subsection{Bacterial Strains, Plasmids, Primers, and Growth Conditions}

Bacterial strains and plasmids used in this study are listed in Table S1. Primers are listed in Table S2. The S. suis 2 strain was cultured in Tryptic Soy Broth (TSB) or on Tryptic Soy Agar (TSA; Difco Laboratories, Detroit, MI, USA), supplemented with $10 \%(v / v)$ newborn bovine serum at $37^{\circ} \mathrm{C}$. E. coli strains were cultured in Luria-Bertani (LB) broth or on LB agar at $37^{\circ} \mathrm{C}$. When required, antibiotics were added at the following concentrations: $75 \mu \mathrm{g} / \mathrm{mL}$ ampicillin or $25 \mu \mathrm{g} / \mathrm{mL}$ kanamycin for E. coli.

\subsection{Bioinformatics Analysis, RNA Isolation and RT-PCR Analysis}

The putative type II TA systems in the S. suis 2 strain SC84 were predicted with TAfinder (http:/ / 202.120.12.133/TAfinder/TAfinder.php). In our work, S. suis 2 was grown to the mid-log-phase and was used to extract the total RNA. The total RNA was purified by using an SV (spin or vacuum) total RNA isolation system (Promega, Madison, WI, USA), according to the manufacturer's protocol. The RNA integrity and concentrations were determined by agarose gel electrophoresis and NanoDrop, respectively. The cDNAs were generated from these RNA samples with HiScript II Q RT SuperMix (Vazyme, Nanjing, China). We used the specific primers (0547F/0548R, 0791F/0790R, 0792F/0791R, 0842F/0841R, 0860F/0861R, 1035F/1034R, 1349F/1348R, and 1821F/1820R) in Table S2 to confirm the co-transcription of putative toxin genes and antitoxin genes.

\subsection{Putative TA Systems Characterized by a Selective Expression Vector}

The pETBAD, a selective expression plasmid, was used to characterize the putative TA systems in E. coli, as previously described [10]. First, each putative antitoxin was amplified from the genome of S. suis 2 using the primer pairs (0547F/R, 0790F/R, 0792F/R, 0842F/R, 0860F/R, 1035F/R, 1349F/R, $1818 \mathrm{~F} / \mathrm{R}$, and $1821 \mathrm{~F} / \mathrm{R}$ ) listed in Table $\mathrm{S} 2$, and the different fragments were digested, using the appropriate restriction enzymes, and cloned into the pETBAD plasmid in order to construct the pETBAD-antitoxin. Then, each putative toxin was amplified from the genome of $S$. suis 2 using the primer pairs $(0548 \mathrm{~F} / \mathrm{R}, 0791 \mathrm{~F} / \mathrm{R}, 0841 \mathrm{~F} / \mathrm{R}, 0861 \mathrm{~F} / \mathrm{R}, 1034 \mathrm{~F} / \mathrm{R}, 1348 \mathrm{~F} / \mathrm{R}, 1817 \mathrm{~F} / \mathrm{R}$, and 1820F/R) listed in Table S2, and they were digested using the appropriate restriction enzymes and cloned into the related, pETBAD-antitoxin, in order to construct the plasmid of the pETBAD-antitoxin-toxin (pETBAD-0547-0548, -0790-0791, -0792-0791, -0842-0841, -0860-0861, -1035-1034, -1349-1348, -1818-1817, and -1821-1820). Each putative toxin was induced by L-arabinose, and its cognate antitoxin was induced by IPTG. E. coli BL21 (DE3) pLysS cells, harboring the corresponding selective expression plasmids, were incubated in LB broth, supplemented with $25 \mu \mathrm{g} / \mathrm{mL}$ kanamycin to $\mathrm{OD}_{600}$ of $0.6-0.8$. Each culture was serially diluted, and $5-\mu \mathrm{L}$ drops, with concentrations ranging from $10^{0}$ (top) to $10^{-5}$ (bottom), were spotted onto four different plates, three of which were provided with various additional concentrations of L-arabinose $(0.025 \%, 0.05 \%, 0.10 \%$, and $0.20 \%), 1 \mathrm{mM}$ IPTG, or both (induction conditions), and the fourth was provided with an additional $0.20 \%$ D-glucose (repression conditions), serving as the control.

\subsection{Toxicity Effect of Each Toxin on E. coli Growth}

Different fragments were amplified from the genome of $S$. suis 2 using the primer pairs (T0548F/R, T0791F/R, T0861F/R, T1034F/R, T1348F/R, and T1820F/R) listed in Table S2. After digesting them using the appropriate restriction enzymes, these fragments were ligated into the pBADhisA, and then the pBADhisA-toxin (pBADhisA-0548, -0791, -0861, -1034, -1348, and -1820) plasmids were constructed. E. coli Top10 cells, into which the related pBADhisA-toxin plasmids were transformed, were grown in LB broth, with an additional $75 \mu \mathrm{g} / \mathrm{mL}$ ampicillin and $0.20 \%$ D-glucose at $37^{\circ} \mathrm{C}$ overnight. The next day, the cultures of the Top10 cells, carrying the plasmids of the pBADhisA-toxin or pBADhisA (control), were diluted at 1:100 in the fresh medium, supplemented with $75 \mu \mathrm{g} / \mathrm{mL}$ ampicillin (LB-ampicillin), and grown to $\mathrm{OD}_{600}$ of $0.05-0.20$. Each culture was then divided into two parts. One half was grown in 
the presence of $0.20 \%$ D-glucose (repression conditions), while the other was grown in the presence of $0.20 \%$ L-arabinose (induction conditions). Culture growth was monitored by measuring $\mathrm{OD}_{600}$ every hour. On the other hand, the cultures were diluted at 1:100 in LB-ampicillin and grown to $\mathrm{OD}_{600}$ of $0.6-0.8$. Then, they were serially diluted, and $5-\mu \mathrm{L}$ drops, with concentrations of $10^{0}$ (top) to $10^{-5}$ (bottom), were successively spotted onto the plates with $0.20 \%$ L-arabinose or $0.20 \%$ D-glucose.

\subsection{Effect of Antitoxin on E. coli Growth}

Different fragments were amplified from the genome of SC19 using the primer pairs (0547F/R, $1349 \mathrm{~F} / \mathrm{R}$, and $1821 \mathrm{~F} / \mathrm{R})$ listed in Table S2. After digesting them using the appropriate restriction enzymes, these fragments were ligated into the pET30a. Then, the plasmids of the pET30a-antitoxin (pET30a-0547, -1349, and -1821) were constructed. E. coli BL21 (DE3) pLysS cells, into which the pBADhisA-0548 and pET30a-0547 (or pET30a), pBADhisA-1348 and pET30a-1349 (or pET30a), and pBADhisA-1820 and pET30a-1821 (or pET30a) were co-transformed, were grown in LB broth, supplemented with $75 \mu \mathrm{g} / \mathrm{mL}$ ampicillin and $25 \mu \mathrm{g} / \mathrm{mL}$ kanamycin at $37{ }^{\circ} \mathrm{C}$ overnight. The next day, the cultures of BL21 (DE3) pLysS cells, carrying the related plasmids of the pBADhisA-toxin and pET30a-antitoxin (or pET30a, as control), were diluted at 1:100 in the fresh medium, supplemented with $75 \mu \mathrm{g} / \mathrm{mL}$ ampicillin and $25 \mu \mathrm{g} / \mathrm{mL}$ kanamycin, and grown to $\mathrm{OD}_{600}$ of $0.2-0.3$. Then, each culture was provided with an additional $1 \mathrm{mM}$ IPTG and $0.20 \%$ L-arabinose, as inducers. Culture growth was monitored by measuring $\mathrm{OD}_{600}$ every hour. During the growth experiments, the samples of E. coli, harboring the related plasmids under induction or un-induction conditions, were collected in vitro at $5 \mathrm{~h}$. Then, E. coli cells were treated using the gram stain. Microscopy images were acquired using a $100 \times$ objective, under oil-immersion.

\subsection{Promoter Activity Assay}

The primer pairs used in this experiment are listed in Table S2. The $260 \mathrm{bp}$ upstream region, preceding the antitoxin ( $r e l B 1$, relB2, or parD) start codon, was selected as their promoter regions. The different fragments (antitoxin', antitoxin-toxin', and antitoxin-toxin) were PCR-amplified. The antitoxin' contained the promoter region and the first $45 \mathrm{bp}$ of the coding region of the antitoxin; the antitoxin-toxin' contained the promoter region, the antitoxin coding region and the first $45 \mathrm{bp}$ coding region of the toxin; and the antitoxin-toxin contained the promoter region and the full-length TA operon. Then, the ribosome-binding site (RBS) was fused to these different fragments. The PCR products were digested with EcoRI and BamHI and cloned into promoter-less lacZ-fusion vector pHGEI01 in order to construct the pHGEI01-antitoxin', pHGEI01-antitoxin-toxin', and pHGEI01-antitoxin-toxin plasmids, and the promoter-less lacZ-fusion vector pHGEI01 was used as the negative control (Figure S3). For the first-step PCR, we used pHGEI01-P0547-0548-F (-P1349-1348-F or -P1821-1820-F) as the forward primes, and pHGEI01-0547'-R1 (-1349'-R1 or -1821'-R1) and pHGEI01-0548'-R1 (-1348'-R1 or $\left.-1820^{\prime}-\mathrm{R} 1\right)$ as the reverse primes, respectively. We then used the primer pairs, pHGEI01-P0547-0548-F (-P1349-1348-F or -P1821-1820-F)/pHGEI01-common-R2, to add the RBS (AGATCTCACACAGGAAACAGCT) sequence between the special genes (antitoxin', antitoxin-toxin', and antitoxin-toxin) and the lacZ gene for the second-step PCR. The pHGEI01-based (-relB1', -relB1-relE1', -relB1-relE1, -relB2', -relB2-relE2', -relB2-relE2, -parD', -parD-parE', and -parD-parE) plasmids were constructed, and the promoter activity assay was performed, as previously described [36]. The pHGEI01-based plasmids were transformed into the E. coli WM3064 strain. WM3064 cells, grown to the mid-exponential phase $\left(\mathrm{OD}_{600} \sim 0.6-0.7\right)$, were collected by centrifugation, washed with PBS, and resuspended in lysis buffer $(0.25 \mathrm{M}$ Tris $/ \mathrm{HCl}, \mathrm{pH} 7.5,0.5 \%$ Trion-X100). Then, the soluble proteins were released by sonication, and the concentrations of the proteins were measured using a bicinchoninic acid (BCA) protein assay kit (Biosharp, Wuhan, China). The $\beta$-galactosidase activity was measured using a $\beta$-galactosidase assay kit (TIANDZ, Beijing, China), according to the manufacturer's instructions [37]. 
Supplementary Materials: The following are available online at http:/ /www.mdpi.com/2072-6651/10/11/467/ s1: Figure S1: Genomic location of nine putative type II toxin-antitoxin (TA) systems in the chromosome of S. suis SC84. Figure S2: Genetic organization of nine putative type II toxin-antitoxin (TA) systems. Figure S3: Schematic representation of the constructed reporter systems for the promoter activity assay. Table S1: Bacterial strains and plasmids used in this study. Table S2: Primers used in this study.

Author Contributions: J.X., H.C. and W.B. conceived and designed the experiments; J.X., N.Z., M.C., S.R., T.Z., M.Q. and X.Z. performed the experiments; J.X., S.R. and F.Y. analyzed the data; J.X. wrote the manuscript.

Funding: This research was supported by the National Natural Science Foundation of China (No. 31672560), the Fundamental Research Funds for the Central Universities (No. 22662018PY042), Competitive planning project from Hubei Academy of Agricultural Sciences (2015jzxjh03), the Natural Science Foundation of Hubei Province (No. 2018CFA045 and No. 2016CFA015) and China Agriculture Research System (CARS-35).

Acknowledgments: We are grateful to Xiaoxue Wang (South China Sea Institute of Oceanology, Chinese Academy of Science, Guangzhou) for providing plasmids pHGEI01 and Escherichia coli WM3064 strain. And we thank Chengkun Zheng (Jiangsu Key Laboratory of Zoonosis, Yangzhou University, Yangzhou) for his technical support.

Conflicts of Interest: The authors declare no conflict of interest.

\section{References}

1. Feng, Y.; Zhang, H.; Ma, Y.; Gao, G.F. Uncovering newly emerging variants of Streptococcus suis, an important zoonotic agent. Trends Microbiol. 2010, 18, 124-131. [CrossRef] [PubMed]

2. Lun, Z.R.; Wang, Q.P.; Chen, X.G.; Li, A.X.; Zhu, X.Q. Streptococcus suis: An emerging zoonotic pathogen. Lancet. Infect. Dis. 2007, 7, 201-209. [CrossRef]

3. Li, Q.; Zhang, Y.H.; Du, D.C.; Yu, Y.F.; Zhang, W. Characterization and functional analysis of PnuC that is involved in the oxidative stress tolerance and virulence of Streptococcus suis serotype 2. Vet. Microbiol. 2018, 216, 198-206. [CrossRef] [PubMed]

4. Gottschalk, M.; Xu, J.; Calzas, C.; Segura, M. Streptococcus suis: A new emerging or an old neglected zoonotic pathogen? Future Microbiol. 2010, 5, 371-391. [CrossRef] [PubMed]

5. Segura, M.; Zheng, H.; de Greeff, A.; Gao, G.F.; Grenier, D.; Jiang, Y.; Lu, C.; Maskell, D.; Oishi, K.; Okura, M.; et al. Latest developments on Streptococcus suis: An emerging zoonotic pathogen: Part 2. Future Microbiol. 2014, 9 , 587-591. [CrossRef] [PubMed]

6. Gottschalk, M.; Segura, M.; Xu, J. Streptococcus suis infections in humans: The Chinese experience and the situation in North America. Anim. Health Res. Rev. 2007, 8, 29-45. [CrossRef] [PubMed]

7. Huong, V.T.; Ha, N.; Huy, N.T.; Horby, P.; Nghia, H.D.; Thiem, V.D.; Zhu, X.; Hoa, N.T.; Hien, T.T.; Zamora, J.; et al. Epidemiology, clinical manifestations, and outcomes of Streptococcus suis infection in humans. Emerg. Infect. Dis. 2014, 20, 1105-1114. [CrossRef] [PubMed]

8. Hill, J.E.; Gottschalk, M.; Brousseau, R.; Harel, J.; Hemmingsen, S.M.; Goh, S.H. Biochemical analysis, cpn60 and 16S rDNA sequence data indicate that Streptococcus suis serotypes 32 and 34, isolated from pigs, are Streptococcus orisratti. Vet. Microbiol. 2005, 107, 63-69. [CrossRef] [PubMed]

9. Tang, J.; Wang, C.; Feng, Y.; Yang, W.; Song, H.; Chen, Z.; Yu, H.; Pan, X.; Zhou, X.; Wang, H.; et al. Streptococcal toxic shock syndrome caused by Streptococcus suis serotype 2. PLoS Med. 2006, 3, e151.

10. Zheng, C.; Xu, J.; Ren, S.; Li, J.; Xia, M.; Chen, H.; Bei, W. Identification and characterization of the chromosomal yefM-yoeB toxin-antitoxin system of Streptococcus suis. Sci. Rep. 2015, 5, 13125. [CrossRef] [PubMed]

11. Yao, X.; Chen, T.; Shen, X.; Zhao, Y.; Wang, M.; Rao, X.; Yin, S.; Wang, J.; Gong, Y.; Lu, S.; et al. The chromosomal SezAT toxin-antitoxin system promotes the maintenance of the SsPI-1 pathogenicity island in epidemic Streptococcus suis. Mol. Microbiol. 2015, 98, 243-257. [CrossRef] [PubMed]

12. Xie, Y.; Wei, Y.; Shen, Y.; Li, X.; Zhou, H.; Tai, C.; Deng, Z.; Ou, H.Y. TADB 2.0: An updated database of bacterial type II toxin-antitoxin loci. Nucleic Acids Res. 2018, 46, D749-D753. [CrossRef] [PubMed]

13. Gerdes, K.; Christensen, S.K.; Lobner-Olesen, A. Prokaryotic toxin-antitoxin stress response loci. Nat. Rev. Microbiol. 2005, 3, 371-382. [CrossRef] [PubMed]

14. Wang, Y.; Wang, H.; Hay, A.J.; Zhong, Z.; Zhu, J.; Kan, B. Functional RelBE-family toxin-antitoxin pairs affect biofilm maturation and intestine colonization in Vibrio cholerae. PLoS ONE 2015, 10, e0135696. [CrossRef] [PubMed] 
15. Kedzierska, B.; Hayes, F. Emerging roles of toxin-antitoxin modules in bacterial pathogenesis. Molecules 2016, 21, 790. [CrossRef] [PubMed]

16. Wen, Y.R.; Behiels, E.; Devreese, B. Toxin-antitoxin systems: Their role in persistence, biofilm formation, and pathogenicity. Pathog. Dis. 2014, 70, 240-249. [CrossRef] [PubMed]

17. Goulard, C.; Langrand, S.; Carniel, E.; Chauvaux, S. The Yersinia pestis chromosome encodes active addiction toxins. J. Bacteriol. 2010, 192, 3669-3677. [CrossRef] [PubMed]

18. Pandey, D.P.; Gerdes, K. Toxin-antitoxin loci are highly abundant in free-living but lost from host-associated prokaryotes. Nucleic Acids Res. 2005, 33, 966-976. [CrossRef] [PubMed]

19. Jorgensen, M.G.; Pandey, D.P.; Jaskolska, M.; Gerdes, K. HicA of Escherichia coli defines a novel family of translation-independent mRNA interferases in bacteria and archaea. J. Bacteriol. 2009, 191, 1191-1199. [CrossRef] [PubMed]

20. Hazan, R.; Sat, B.; Engelberg-Kulka, H. Escherichia coli mazEF-mediated cell death is triggered by various stressful conditions. J. Bacteriol. 2004, 186, 3663-3669. [CrossRef] [PubMed]

21. Nigam, A.; Kumar, S.; Engelberg-Kulka, H. Quorum sensing extracellular death peptides enhance the endoribonucleolytic activities of Mycobacterium tuberculosis MazF toxins. mBio 2018, 9. [CrossRef] [PubMed]

22. Schuster, C.F.; Park, J.H.; Prax, M.; Herbig, A.; Nieselt, K.; Rosenstein, R.; Inouye, M.; Bertram, R. Characterization of a mazEF toxin-antitoxin homologue from Staphylococcus equorum. J. Bacteriol. 2013, 195, 115-125. [CrossRef] [PubMed]

23. Syed, M.A.; Koyanagi, S.; Sharma, E.; Jobin, M.C.; Yakunin, A.F.; Levesque, C.M. The chromosomal mazEF locus of Streptococcus mutans encodes a functional type II toxin-antitoxin addiction system. J. Bacteriol. 2011, 193, 1122-1130. [CrossRef] [PubMed]

24. Yuan, J.; Yamaichi, Y.; Waldor, M.K. The three Vibrio cholerae chromosome II-encoded ParE toxins degrade chromosome I following loss of chromosome II. J. Bacteriol. 2011, 193, 611-619. [CrossRef] [PubMed]

25. Christensen-Dalsgaard, M.; Gerdes, K. Two higBA loci in the Vibrio cholerae superintegron encode mRNA cleaving enzymes and can stabilize plasmids. Mol. Microbiol. 2006, 62, 397-411. [CrossRef] [PubMed]

26. Iqbal, N.; Guerout, A.M.; Krin, E.; Le Roux, F.; Mazel, D. Comprehensive functional analysis of the 18 Vibrio cholerae N16961 toxin-antitoxin systems substantiates their role in stabilizing the superintegron. J. Bacteriol. 2015, 197, 2150-2159. [CrossRef] [PubMed]

27. Gupta, A.; Venkataraman, B.; Vasudevan, M.; Gopinath Bankar, K. Co-expression network analysis of toxin-antitoxin loci in Mycobacterium tuberculosis reveals key modulators of cellular stress. Sci. Rep. 2017, 7, 5868. [CrossRef] [PubMed]

28. Frampton, R.; Aggio, R.B.; Villas-Boas, S.G.; Arcus, V.L.; Cook, G.M. Toxin-antitoxin systems of Mycobacterium smegmatis are essential for cell survival. J. Biol. Chem. 2012, 287, 5340-5356. [CrossRef] [PubMed]

29. De la Cruz, M.A.; Zhao, W.; Farenc, C.; Gimenez, G.; Raoult, D.; Cambillau, C.; Gorvel, J.P.; Meresse, S. A toxin-antitoxin module of Salmonella promotes virulence in mice. PLoS Pathog. 2013, 9, e1003827. [CrossRef] [PubMed]

30. Komi, K.K.; Ge, Y.M.; Xin, X.Y.; Ojcius, D.M.; Sun, D.; Hu, W.L.; Zhao, X.; Lin, X.; Yan, J. Chpk and MazF of the toxin-antitoxin modules are involved in the virulence of Leptospira interrogans during infection. Microbes Infect. 2015, 17, 34-47. [CrossRef] [PubMed]

31. Schneider, B.; Weigel, W.; Sztukowska, M.; Demuth, D.R. Identification and functional characterization of type II toxin/antitoxin systems in Aggregatibacter actinomycetemcomitans. Mol. Oral Microbiol. 2018, 33, 224-233. [CrossRef] [PubMed]

32. Chan, W.T.; Espinosa, M.; Yeo, C.C. Keeping the wolves at bay: Antitoxins of prokaryotic type II toxin-antitoxin systems. Front. Mol. Biosci. 2016, 3, 9. [CrossRef] [PubMed]

33. Jiang, Y.; Pogliano, J.; Helinski, D.R.; Konieczny, I. ParE toxin encoded by the broad-host-range plasmid RK2 is an inhibitor of Escherichia coli gyrase. Mol. Microbiol. 2002, 44, 971-979. [CrossRef] [PubMed]

34. Fiebig, A.; Castro Rojas, C.M.; Siegal-Gaskins, D.; Crosson, S. Interaction specificity, toxicity and regulation of a paralogous set of ParE/RelE-family toxin-antitoxin systems. Mol. Microbiol. 2010, 77, 236-251. [CrossRef] [PubMed]

35. Yamaguchi, Y.; Inouye, M. Regulation of growth and death in Escherichia coli by toxin-antitoxin systems. Nat. Rev. Microbiol. 2011, 9, 779-790. [CrossRef] [PubMed]

36. Guo, Y.; Yao, J.; Sun, C.; Wen, Z.; Wang, X. Characterization of the deep-sea Streptomyces sp. SCSIO 02999 derived VapC/VapB toxin-antitoxin system in Escherichia coli. Toxins 2016, 8, 195. [CrossRef] [PubMed] 
37. Zheng, C.; Zhao, X.; Zeng, T.; Cao, M.; Xu, J.; Shi, G.; Li, J.; Chen, H.; Bei, W. Identification of four type II toxin-antitoxin systems in Actinobacillus pleuropneumoniae. FEMS Microbiol. Lett. 2017, 364. [CrossRef] [PubMed]

38. Syed, M.A.; Levesque, C.M. Chromosomal bacterial type II toxin-antitoxin systems. Can. J. Microbiol. 2012, 58, 553-562. [CrossRef] [PubMed]

39. Li, G.; Shen, M.; Lu, S.; Le, S.; Tan, Y.; Wang, J.; Zhao, X.; Shen, W.; Guo, K.; Yang, Y.; et al. Identification and characterization of the Hic $\mathrm{AB}$ toxin-antitoxin system in the opportunistic pathogen Pseudomonas aeruginosa. Toxins 2016, 8, 113. [CrossRef] [PubMed]

40. Lee, I.G.; Lee, S.J.; Chae, S.; Lee, K.Y.; Kim, J.H.; Lee, B.J. Structural and functional studies of the Mycobacterium tuberculosis VapBC30 toxin-antitoxin system: Implications for the design of novel antimicrobial peptides. Nucleic Acids Res. 2015, 43, 7624-7637. [CrossRef] [PubMed]

41. Kang, S.M.; Kim, D.H.; Lee, K.Y.; Park, S.J.; Yoon, H.J.; Lee, S.J.; Im, H.; Lee, B.J. Functional details of the Mycobacterium tuberculosis VapBC26 toxin-antitoxin system based on a structural study: Insights into unique binding and antibiotic peptides. Nucleic Acids Res. 2017, 45, 8564-8580. [CrossRef] [PubMed]

42. Gotfredsen, M.; Gerdes, K. The Escherichia coli relBE genes belong to a new toxin-antitoxin gene family. Mol. Microbiol. 1998, 29, 1065-1076. [CrossRef] [PubMed]

43. Chan, W.T.; Nieto, C.; Harikrishna, J.A.; Khoo, S.K.; Othman, R.Y.; Espinosa, M.; Yeo, C.C. Genetic regulation of the yefM-yoeB toxin-antitoxin locus of Streptococcus pneumoniae. J. Bacteriol. 2011, 193, 4612-4625. [CrossRef] [PubMed]

44. Yoshizumi, S.; Zhang, Y.; Yamaguchi, Y.; Chen, L.; Kreiswirth, B.N.; Inouye, M. Staphylococcus aureus YoeB homologues inhibit translation initiation. J. Bacteriol. 2009, 191, 5868-5872. [CrossRef] [PubMed]

45. Larson, A.S.; Hergenrother, P.J. Light activation of Staphylococcus aureus toxin YoeBSa1 reveals guanosinespecific endoribonuclease activity. Biochemistry 2014, 53, 188-201. [CrossRef] [PubMed]

46. Khoo, S.K.; Loll, B.; Chan, W.T.; Shoeman, R.L.; Ngoo, L.; Yeo, C.C.; Meinhart, A. Molecular and structural characterization of the PezAT chromosomal toxin-antitoxin system of the human pathogen Streptococcus pneumoniae. J. Biol. Chem. 2007, 282, 19606-19618. [CrossRef] [PubMed]

47. Mutschler, H.; Reinstein, J.; Meinhart, A. Assembly dynamics and stability of the pneumococcal epsilon zeta antitoxin toxin (PezAT) system from Streptococcus pneumoniae. J. Biol. Chem. 2010, 285, 21797-21806. [CrossRef] [PubMed]

48. Chan, W.T.; Espinosa, M. The Streptococcus pneumoniae pezAT toxin-antitoxin system reduces beta-lactam resistance and genetic competence. Front. Microbiol. 2016, 7, 1322. [CrossRef] [PubMed]

49. Korch, S.B.; Contreras, H.; Clark-Curtiss, J.E. Three Mycobacterium tuberculosis Rel toxin-antitoxin modules inhibit mycobacterial growth and are expressed in infected human macrophages. J. Bacteriol. 2009, 191, 1618-1630. [CrossRef] [PubMed]

50. Nieto, C.; Pellicer, T.; Balsa, D.; Christensen, S.K.; Gerdes, K.; Espinosa, M. The chromosomal relBE2 toxin-antitoxin locus of Streptococcus pneumoniae: Characterization and use of a bioluminescence resonance energy transfer assay to detect toxin-antitoxin interaction. Mol. Microbiol. 2006, 59, 1280-1296. [CrossRef] [PubMed]

51. Chan, W.T.; Yeo, C.C.; Sadowy, E.; Espinosa, M. Functional validation of putative toxin-antitoxin genes from the Gram-positive pathogen streptococcus pneumoniae: Phd-doc is the fourth bona-fide operon. Front. Microbiol. 2014, 5, 677. [CrossRef] [PubMed]

52. Kim, D.H.; Kang, S.M.; Park, S.J.; Jin, C.; Yoon, H.J.; Lee, B.J. Functional insights into the Streptococcus pneumoniae HicBA toxin-antitoxin system based on a structural study. Nucleic Acids Res. 2018, 46, 6371-6386. [CrossRef] [PubMed]

53. Christensen, S.K.; Gerdes, K. RelE toxins from bacteria and archaea cleave mRNAs on translating ribosomes, which are rescued by tmRNA. Mol. Microbiol. 2003, 48, 1389-1400. [CrossRef] [PubMed]

54. Pedersen, K.; Zavialov, A.V.; Pavlov, M.Y.; Elf, J.; Gerdes, K.; Ehrenberg, M. The bacterial toxin RelE displays codon-specific cleavage of mRNAs in the ribosomal A site. Cell 2003, 112, 131-140. [CrossRef]

55. Christensen-Dalsgaard, M.; Jorgensen, M.G.; Gerdes, K. Three new RelE-homologous mRNA interferases of Escherichia coli differentially induced by environmental stresses. Mol. Microbiol. 2010, 75, 333-348. [CrossRef] [PubMed] 
56. Unterholzner, S.J.; Hailer, B.; Poppenberger, B.; Rozhon, W. Characterisation of the stbD/E toxin-antitoxin system of pEP36, a plasmid of the plant pathogen Erwinia pyrifoliae. Plasmid 2013, 70, 216-225. [CrossRef] [PubMed]

57. Davis, T.L.; Helinski, D.R.; Roberts, R.C. Transcription and autoregulation of the stabilizing functions of broad-host-range plasmid RK2 in Escherichia coli, Agrobacterium tumefaciens and Pseudomonas aeruginosa. Mol. Microbiol. 1992, 6, 1981-1994. [CrossRef] [PubMed]

58. Gupta, M.; Nayyar, N.; Chawla, M.; Sitaraman, R.; Bhatnagar, R.; Banerjee, N. The chromosomal parDE2 toxin-antitoxin system of Mycobacterium tuberculosis H37Rv: Genetic and functional characterization. Front. Microbiol. 2016, 7, 886. [CrossRef] [PubMed]

59. Yuan, J.; Sterckx, Y.; Mitchenall, L.A.; Maxwell, A.; Loris, R.; Waldor, M.K. Vibrio cholerae ParE2 poisons DNA gyrase via a mechanism distinct from other gyrase inhibitors. J. Biol. Chem. 2010, 285, 40397-40408. [CrossRef] [PubMed]

60. Li, G.Y.; Zhang, Y.; Inouye, M.; Ikura, M. Structural mechanism of transcriptional autorepression of the Escherichia coli RelB/RelE antitoxin/toxin module. J. Mol. Biol. 2008, 380, 107-119. [CrossRef] [PubMed]

61. Li, P.; Tai, C.; Deng, Z.; Gan, J.; Oggioni, M.R.; Ou, H.Y. Identification and characterization of chromosomal relBE toxin-antitoxin locus in Streptomyces cattleya DSM46488. Sci. Rep. 2016, 6, 32047. [CrossRef] [PubMed]

62. Zhao, J.L.; Liu, W.; Xie, W.Y.; Cao, X.D.; Yuan, L. Viability, biofilm formation, and MazEF expression in drug-sensitive and drug-resistant Mycobacterium tuberculosis strains circulating in Xinjiang, China. Infect. Drug Resist. 2018, 11, 345-358. [CrossRef] [PubMed]

63. Wang, X.Y.; Zhao, X.K.; Wang, H.; Huang, X.; Duan, X.K.; Gu, Y.Z.; Lambert, N.; Zhang, K.; Kou, Z.H.; Xie, J.P. Mycobacterium tuberculosis toxin Rv2872 is an RNase involved in vancomycin stress response and biofilm development. Appl. Microbiol. Biot. 2018, 102, 7123-7133. [CrossRef] [PubMed]

64. Chan, W.T.; Domenech, M.; Moreno-Cordoba, I.; Navarro-Martinez, V.; Nieto, C.; Moscoso, M.; Garcia, E.; Espinosa, M. The Streptococcus pneumoniae yefM-yoeB and relBE toxin-antitoxin operons participate in oxidative stress and biofilm formation. Toxins 2018, 10, 378. [CrossRef] [PubMed] 